\author{
GIANCARLO RAPPAZZO $(*)\left(^{\circ}\right)$ - ELIANA SALVO $(*)$ - EUSTACHIO TARASCO $(* * *)$ \\ GIULIO PETRONIO PETRONIO $(* *)$ - MARIA ANTONIETTA BUCCHERI $(*)$ - PIO MARIA FURNERI $(* *)$ \\ VIRGINIA FUOCHI $(* *)$ - MIRELLA CLAUSI $(*)$
}

\title{
ENDOSYMBIONTS OF ENTOMOPATHOGENIC NEMATODES FROM SOUTH ITALY: A PHENOTYPIC STUDY
}

\author{
(*) Dept. of Biological Geological Environmental Sciences; \\ (**) Dept. of Biomedical and Biotechnological Sciences BIOMETEC, University of Catania; \\ (***) Dept. of Soil Plant and Food Sciences, University of Bari “A. Moro”, Italy \\ $\left.{ }^{\circ}\right)$ Corresponding Author: rappazzo@unict.it
}

\begin{abstract}
Rappazzo G., Salvo E., Tarasco E., Petronio Petronio G., Buccheri M.A., Furneri P.M., Fuochi V., Clausi M. - Endosymbionts of Entomopathogenic Nematodes from South Italy: a phenotypic study.

We examined different Xenorhabdus strains (five of X. bovienii and two of X. kozodoii), obtained from EPN isolates belonging to the genus Steinernema (S. feltiae, S. ichnusae, S. apuliae, S. vulcanicum) of different geographic origin by both genotypic and phenotypic analysis. Common laboratory assays were done for traits such as antibiotic resistance, haemolytic activity, lactose utilisation, biofilm production, chosen as the least selectable traits for EPN lifecycle, and thus as (presumably) neutral traits. As selective marker, the activity of the endosymbiont's toxins was verified in an in vivo assay on G. mellonella larvae. Genotyping done by $16 \mathrm{~S}$ partial sequencing was used for identification purposes. Xenorhabdus bovienii isolates showed a broad phenotypic spectrum; on the other hand, X. kozodoii showed a less degree of phenotypic variation, reduced ability of biofilm production and conspicuous $\beta$-galactosidase activity. However, all the strains were able to kill G. mellonella larvae with high efficiency.
\end{abstract}

KEY Words: symbiosis, Steinernema, Xenorhabdus, Italy.

\section{INTRODUCTION}

Entomopathogenic Nematodes (EPNs) rely on their endosymbionts for a number of processes which are finalized to kill the insect preys, mostly larvae. However, those endosymbionts are indeed autonomous microorganisms, able to grow in synthetic media, and several have been characterized both biochemically and physiologically (PoINAR, 1990). Furthermore, the existence of an evolutionary link, or coevolution, between the two organisms have been postulated, since each bacterial species is associated with a reduced number of EPN species (ADAMS et al., 2007). For example, Xenorhabdus bovienii was found to be associated with Steinernema feltiae, S. kraussei, S. ichnusae and with some other EPNs of the "feltiae group"; while X. kozodoii was found in association with a number of EPN species from the "glaseri group"; other endosymbionts are similarly specific to other groups of Steinernema (TAILlEZ et al., 2006). Because of this exclusive relationship, endosymbionts are expected to perform their life cycle only within their host, with little (if any) exchange with the environment and/or other organisms, excepting of course the EPN preys (CLAUSI et al., 2012). The presence of such a confined life cycle, if demonstrated, would be of considerable evolutionary interest.

In the present work, we analysed endosymbionts for a number of phenotypic traits which were apparently unrelated to EPNs life cycle and thus possibly of neutral value from an evolutionary point of view. Among them, antibiotic resistance/susceptibility, biofilm production, $\beta$ galactosidase activity seemed the most appropriate for our goal and are also easily performed in laboratory. On the other hand, endosymbionts produce a toxin, or a number of them, which are able to kill the EPN prey thus facilitating the development and the life-cycle of the EPN host (PoINAR, 1979; Clausi et al., 2014). Toxin(s) production is to be considered as a trait of selective value, since it positively increases the fitness of EPN host (HINCHLIFFE et $a l ., 2010)$. It is expected that neutral characters may broadly vary among samples, in particular as long as a closed life cycle takes place, while selective ones should be highly convergent.

To this purpose, five strains of $X$. bovienii and two of $X$. kozodoii, obtained from already described Steinernema isolates from South Italy (TARASCO et al., 2015), were grown in vitro and subjected to a number of biochemical assays originally developed for bacteria of the family Enterobacteriaceae.

\section{MATERIALS AND METHODS}

\section{EPN ISOLATES AND THEIR ENDOSYMBIONTS}

The origin of EPN isolates, all collected from South Italy, including Sardinia and Sicily, has been described (TARASCO et al., 2008; TARASCO et al., 2015; ClAUSI et al., 2011; DE LuCA et al., 2015). The relevant data are detailed in Table 1. EPNs were grown and collected as described in TARASCO et al. (2015).

\section{ISOLATION OF ENDOSYMBIONTS FROM EPNS}

Up to one hundred of freshly collected EPNs were sterilized in 1\% Hyamine 1622 (Sigma-Aldrich) and homogenized. The resulting suspension was serially diluted and aliquots were plated on McConkey Agar (MC) (Oxoid limited) [Peptone $20 \mathrm{~g} / \mathrm{l}$; lactose $10 \mathrm{~g} / \mathrm{l}$; bile salts $5 \mathrm{~g} / \mathrm{l}$; 
Table 1 - EPN isolates and their endosymbionts used in the present study.

\begin{tabular}{|c|c|c|c|c|}
\hline \hline Isolate & EPN species & Area & ITS1 Acc. number & Endosymbiont \\
\hline VE01 & S. feltiae & South Sicily & HQ412835.1 & X. bovienii \\
\hline ESA & S. feltiae & East Sicily & GU599911.1 & X. bovienii \\
\hline CT036 & S. feltiae & East Sicily & n.a. & X. bovienii \\
\hline SAR6 & S. ichnusae & Sardinia & EU421129 & X. bovienii \\
\hline MU1 & S. ichnusae & Campania & HQ412841.1 & X. bovienii \\
\hline CS3 & S. apuliae & Apulia & HQ416968.1 & X. kozodoii \\
\hline Esc1 & S. vulcanicum & East Sicily & GU929442.1 & X. kozodoii \\
\hline
\end{tabular}

sodium chloride $5 \mathrm{~g} / \mathrm{l}$; neutral red $0.075 \mathrm{~g} / \mathrm{l}$; agar $12 \mathrm{~g} / \mathrm{l}$; final $\mathrm{pH} 7.4 \pm 0.2$ ] plates. Growth was allowed at $28^{\circ} \mathrm{C}$ for up to $48 \mathrm{hrs}$. Identification was routinely confirmed on NBTA agar plates (KOPPENHOFER, 2007).

\section{ANTIBIOTIC SUSCEPTIBILITY}

Patterns of antibiotic susceptibility to $\beta$-lactams (penicillin, amoxicillin, cephalothin, cefuroxime, and cefditoren), macrolides and lincosamides (erythromycin and clindamycin), tetracycline, aminoglycosides (gentamicin), fluoroquinolones (ciprofloxacin), glycopeptides (vancomycin) and phenicols (chloramphenicol) were determined by disc diffusion test according to CLSI guidelines (CLSI, 2017). Antibiotic disks were purchased from Oxoid limited.

HeMolytic ACTIVITY, CATALASE ACTIVITY, OXIDASE ACTIVIY AND BIOFILM PRODUCTION

Hemolytic activity was investigated by using Columbia agar base (Oxoid limited) [special peptone $23.0 \mathrm{~g} / 1$; starch $1.0 \mathrm{~g} / \mathrm{l}$; sodium chloride $5 \mathrm{~g} / \mathrm{l}$; agar $10.0 \mathrm{~g} / \mathrm{l}$; final $\mathrm{pH} 7.3 \pm$ 0.2 at $25^{\circ} \mathrm{C}$ ] supplemented by $5 \%$ defibrinated horse blood (Oxoid limited). All strains were incubated aerobically for $24 \mathrm{hrs}$ and then observed for hemolysis production and results as recorded: alpha hemolysis (incomplete haemolytic activity with a not transparent halo), beta hemolysis (sharp and complete haemolytic activity around the colony), gamma hemolysis as no haemolytic activity.

Catalase activity was determined on colonies growth in $\mathrm{MH}$ agar. Briefly, by using a sterile plastic loop, one or two colonies were transferred on a surface of a clean and dry glass slide, then a drop of $3 \%$ hydrogen peroxide was added, and positivity was recorded as bubbling in at least 5 seconds.

Oxidase activity was assessed by using Oxidase Detection Strips (Oxoid limited) according to the procedure suggested by manufacturer.

The assay for in vitro biofilm formation was performed in polystyrene 96 wells microplates (GIUMMARRA et al., 2010) after aerobic incubation for $18-24 \mathrm{hrs}$ at $30^{\circ} \mathrm{C}$. The biofilm index (B.I.) was calculated using the formula: OD570 / OD600 * 0.4. A cut-off value of $0.061 \mathrm{OD}$ was used, and strains were classified as non-producing $(\mathrm{OD}<0.061)$, weak producer $(0.061<\mathrm{OD}<0.120)$, medium-sized producers $(0.121<\mathrm{OD}<0.300)$, strong producer $(\mathrm{OD}>0.300)$. Tests were performed on Muller Hinton broth (MH) (BBL - BD) [Casein acid hydrolysate $1705 \mathrm{~g} / 1$; beef extract $3.0 \mathrm{~g} / 1$; starch $1.5 \mathrm{~g} / \mathrm{l}$; final $\mathrm{pH} 7.3 \pm 0.2$ ] alone or supplemented with $1 \%$ glucose (Sigma-Aldrich), mannose (SigmaAldrich) or sorbitol (Sigma-Aldrich). $\beta$-galactosidase assay was performed following Miller's protocol (MACKAY et al, 1970; FUOCHI et al., 2017).

\section{PARTIAL PURIFICATION OF BACTERIAL TOXIN}

AND IN VIVO PATHOGENICITY ASSAY

The method devised by BRILLARD et al. (2001) was thoroughly followed in order to obtain a protein precipitate cointaining crude (pre-purified) toxins. The whole bacterial culture proteins (not only toxins) are precipitated with this method. Xenorhabdus strains were grown at saturation in LB [tryptone (BD) $10 \mathrm{~g} / \mathrm{l}$; yeast extract (BD) $5 \mathrm{~g} / \mathrm{l} ; \mathrm{NaCl}$ (Sigma-Aldrich) $10 \mathrm{~g} / \mathrm{l}$; final $\mathrm{pH} 7.0]$ broth at $28^{\circ} \mathrm{C}$. The crude post-growth broth, containing the bacterial toxins, was concentrated by two-steps ammonium sulfate (SigmaAldrich) precipitation; the final pellet was resuspended in PBS (one hundredth of the original colture volume) and dialyzed overnight against phosphate buffered saline (PBS) [NaCl (Sigma-Aldrich) $8 \mathrm{~g} / \mathrm{l} ; \mathrm{KCl}$ (Sigma-Aldrich) $0.2 \mathrm{~g} / \mathrm{l}$; $\mathrm{Na}_{2} \mathrm{HPO}_{4}$ (Sigma-Aldrich) $1.44 \mathrm{~g} / \mathrm{l} ; \mathrm{KH}_{2} \mathrm{PO}_{4}$ (SigmaAldrich) $0.24 \mathrm{~g} / \mathrm{l}$; final $\mathrm{pH} 7.4$ by using $\mathrm{HCl}$ (SigmaAldrich)]. Five $\mu 1$ of the so obtained crude toxins were injected into living Galleria mellonella larvae footpads using an Ethanol-sterilized Hamilton microsyringe. Four larvae were used for every bacterial strain; 8 control larvae were given PBS alone.

\section{GENOMIC DNA EXTRACTION AND 16S RRNA \\ GENE AMPLIFICATION}

Genomic DNA was extracted and part of the 16S rRNA gene PCR amplified using primer pairs Xeno_F and Xeno L (TAILlEZ et al., 2006), using conditions described therein. For sequence analysis, the region around positions 950 - 990 of $16 \mathrm{~S}$ rDNA, where diagnostic polymorphisms are found, was amplified with primers Xeno 888 (TGGAGCATGTGGTTTAATTCG) and Xeno 1016 (AACCCAACATTTCACAACACG). Sequence analysis was carried out with Big Dye Terminator 1.1 Sequencing Standard kit on a ABIPRISM 3130 (both from Applied Biosystems).

\section{StATISTICAL ANALYSIS}

Principal component analysis was performed using the software SIMCA v. 13. Cluster analysis was done using dist and hclust functions within the R package.

\section{RESULTS}

\section{GENOTYPING}

16S rDNA sequencing was used to confirm species' attribution, and no intraspecific variations were found with respect to known sequences.

\section{PHENOTYPIC MARKERS}

Xenorhabdus kozodoii grows well on MC plates, forming deep red colonies. In contrast, $X$. bovienii grows irregularly, 
giving rise to different kinds of red-brownish colonies. $\beta$ galactosidase activity was undetectable in $X$. bovienii, but significant in $X$. kozodoii, even if about a half than E. coli ATCC 35218 used as control. Growth on NTBA plates allowed easy differentiation of $X$. bovienii and X. kozodoii because of different use of the chromogenic substrate for glucosidase activity. All strains showed aerobic alphahemolysis on Columbia agar blood plates (Table 2).

The antibiotic susceptibility/resistance spectrum was very composite. All strains were found resistant to penicillins, macrolides, liconsamides glicopeptides, while susceptible to quinolones, tetracyclines, aminoglicosides. However, resistance to cephalosporins was strain dependent. All strains were resistant to cephalothin, while the susceptibility to cefuroxime was strain-dependent: all strains of $X$. kozodoii and one of $X$. bovienii (MU1) were susceptible, while the others were resistant. All strains tested were susceptible to ceftidoren (Table 3).
Biofilm production test showed different B.I. according to Xenorhabdus species and media tested (Fig. I). In MH, both $X$. kozodoii strains were almost non-producing (B.I. 0.041), while $X$. bovienii strains were overall medium-strong producers. Addition of sugars, as expected, caused marked B.I. increase, so that $X$. kozodoii showed a tenfold increase, while $X$. bovienii switched from small to moderate.

\section{TOXICITY OF CRUDE PROTEIN EXTRACT}

ON G. MELLONELLA LARVAE

The protein extract showed similar activity both on $X$. bovienii and on X. kozodoii, resulting in rapid (within 24 hrs) death of insect larvae at the dose of $5 \mu \mathrm{l}$; some dead even within $12 \mathrm{hrs}$ (Table 4). At this time, no significant differences were found among strains or between $X$. bovienii and X. kozodoii. Interestingly, larvae became blackish in the same way as when infected by EPNs, even if, as expected, no massive bacterial infection was seen, but

Table 2 - Distinctive features of endosymbiont growth in selected conditions.

\begin{tabular}{|c|c|c|c|c|c|}
\hline \hline Strains & McConkey & Blood agar & Catalase & Oxidase & $\beta$-galactosidase \\
\hline VE01 & brown & $\alpha$-hemolytic & Negative & negative & negative \\
\hline ESA & brown & $\alpha$-hemolytic & Negative & negative & negative \\
\hline CT036 & brown & $\alpha$-hemolytic & Negative & negative & negative \\
\hline SAR6 & brown & $\alpha$-hemolytic & Negative & negative & negative \\
\hline MU1 & brown & $\alpha$-hemolytic & Negative & negative & negative \\
\hline ESC1 & red & $\alpha$-hemolytic & Negative & negative & positive \\
\hline CS3 & red & $\alpha$-hemolytic & Negative & negative & positive \\
\hline
\end{tabular}

Table 3 - Pattern of antibiotic resistance/susceptibility (inhibition halo in $\mathrm{mm}$ ).

\begin{tabular}{|c|c|c|c|c|c|c|c|c|c|}
\hline Strains & Penicillin & Amoxicillin & Cefuroxime & Erythromycin & Clindamycin & Tetracycline & Ciprofloxacin & Gentamycin & Chloramphenicol \\
\hline VE01 & n.h* & $\mathrm{n} . \mathrm{h}^{*}$ & $\mathrm{n} . \mathrm{h}^{*}$ & n.h* & n.h* & 35 & 45 & 33 & 48 \\
\hline ESA & n.h* & n.h* & 11 & 9 & n.h* & 33 & 40 & 25 & 37 \\
\hline СТ036 & n.h* & n.h* & 12 & n.h* & n.h* & 34 & 40 & 23 & 43 \\
\hline SAR6 & $\mathrm{n} \cdot \mathrm{h}^{*}$ & n.h* & 11 & n.h* & n.h* & 30 & 40 & 30 & 42 \\
\hline MU1 & n.h* & n.h* & 18 & 13 & n.h* & 32 & 51 & 30 & 46 \\
\hline $\mathrm{ESC} 1$ & n.h* & n.h* & 21 & n.h* & n.h* & 22 & 26 & 22 & 30 \\
\hline $\mathrm{CS} 3$ & n.h* & n.h* & 14 & n.h* & n.h* & 35 & 32 & 26 & 30 \\
\hline
\end{tabular}

*n.h.: no inhibition halo reported

Fig. I - Biofilm index of different Xenorhabdus spp. Tested media are indicated on the right.

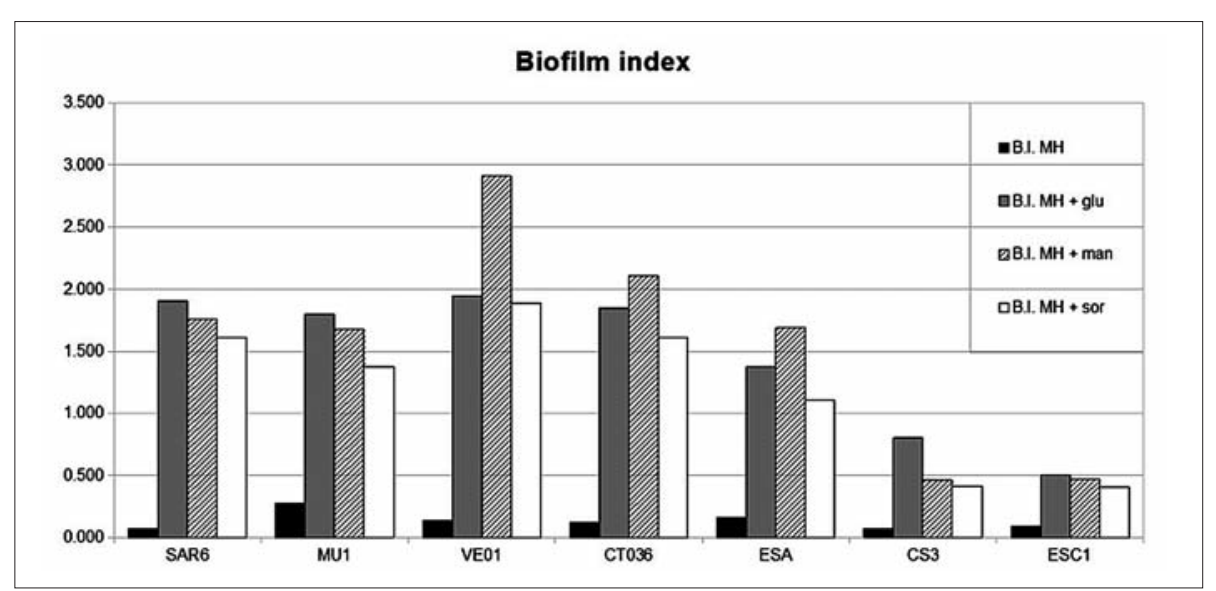


Table 4 - Toxicity of the crude toxin fraction at the time point indicated (n.rs of dead larvae).

\begin{tabular}{|l|c|c|}
\hline \hline Strain & Death at $12 \mathrm{hrs}$ & Death at $24 \mathrm{hrs}$ \\
\hline CT036 & $75 \%$ & $100 \%$ \\
\hline ESA & $25 \%$ & $100 \%$ \\
\hline SAR6 & 0 & $100 \%$ \\
\hline VE01 & 0 & $100 \%$ \\
\hline MU1 & 0 & $100 \%$ \\
\hline ESC1 & 0 & $100 \%$ \\
\hline CS3 & 0 & $100 \%$ \\
\hline Control & 0 & $0 \%$ \\
\hline
\end{tabular}

an important degeneration of internal organs was found (unpublished data).

\section{Principal Component Analysis}

Principal Component Analysis (PCA) provides an understanding of the relationships among the variables, i.e. which variables contribute similar information to the PCA model, and which provide unique information about the observations.

PCA was done on a dataset constructed in such a way as including data of antibiotic susceptibility/resistance, biofilm production, virulence at $12 \mathrm{hrs}$. The first component explained about $55 \%$ of the variance found, the second about $17 \%$; taken together, more than $72 \%$ of the entire variance could be explained by combining both components. The Scatter Plot for the first components is shown in Fig. II. While $X$. kozodoii strains were found close to each other on the left, the other strains, belonging to $X$. bovienii, spread on the right, with no clear evidence of clustering or differentiation among SAR6 and MU1 ( $S$. ichnusae) from the others (S. feltiae). The contribution plot (Fig. III) shows the weight of each variable to the observed distribution. It is apparent that distribution is mainly led by such variables as biofilm production induced by sugars. The effects of antibiotics, with the exception of chloramphenicol, and the pathogenicity (or virulence) seems to play a poorer role in strains' distribution. Finally, hierarchical clustering (Fig. IV) confirmed that strains of $X$.
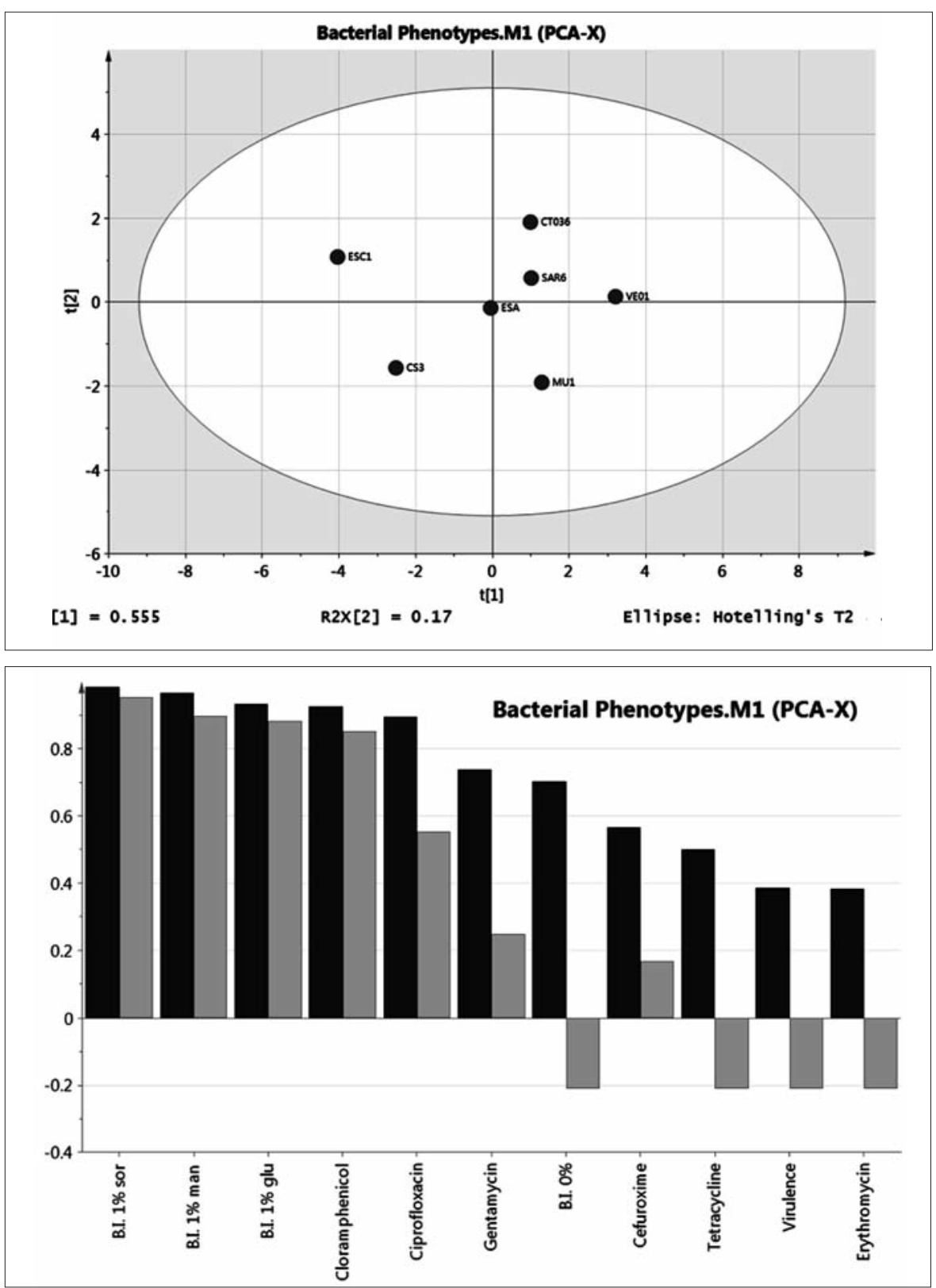

Fig. II - Scatter plot of the strains' variables displaying how the strains' variables are located, in a first-to-second components graph, with respect to each other.
Fig. III - Cumulative R (black) and $\mathrm{Q}$ (grey) for each variable. $\mathrm{R}$ is a measure of fit, i.e. how well the model fits the data; $\mathrm{Q}$ explains how well the model predicts the variable. Acceptable values are $>80 \%$. 
Fig. IV - Cluster analysis of the strains used for the study, based on the variables indicated on the right.

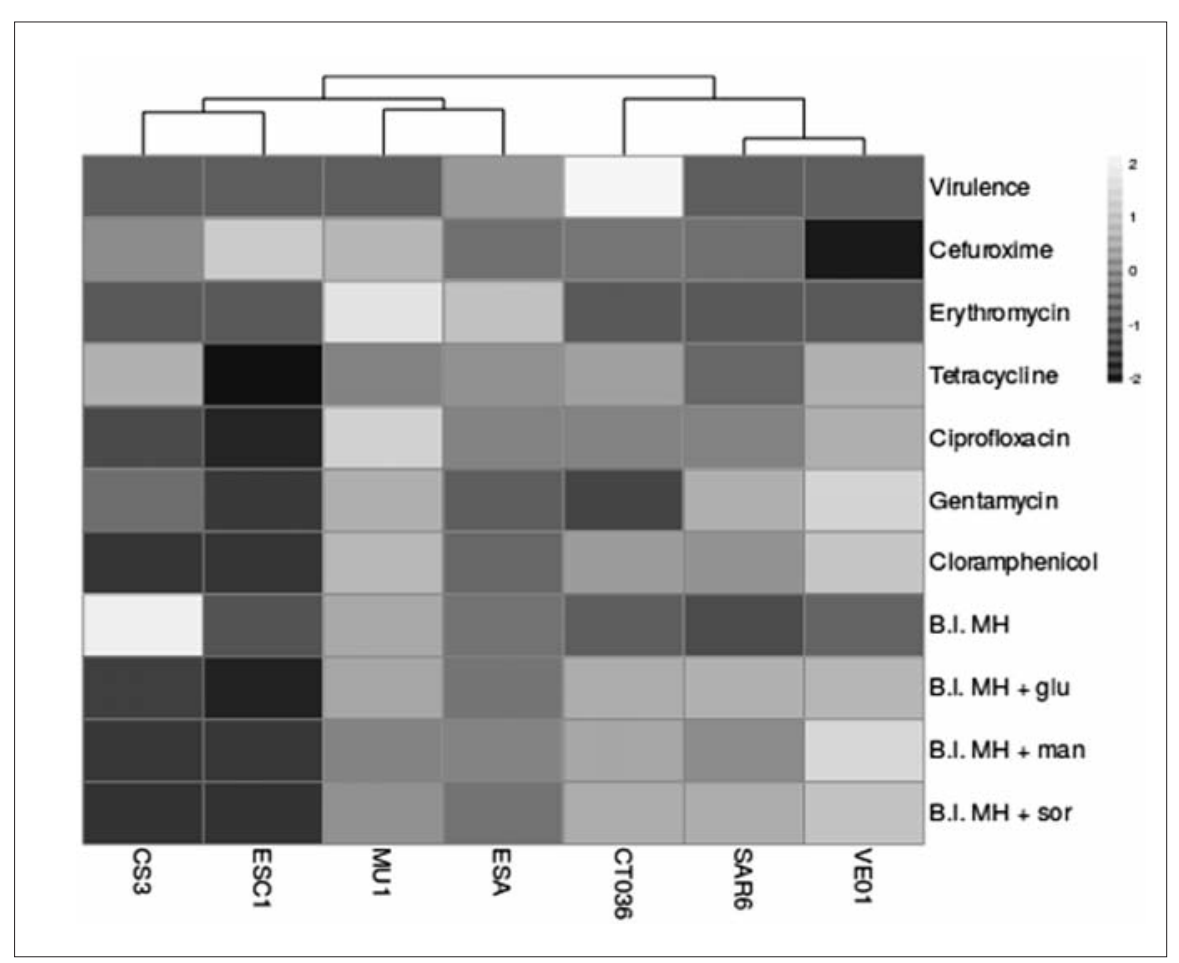

bovienii from $S$. feltiae were intermingled with those obtained from $S$. ichnusae, wile the strains of $X$. kozodoii cluster together. The graph shown in Fig. IV is representative of a number of different assays performed using several clustering algorithms; however, none of them was able to clusterize SAR6 with MU1 and differentiate them from $X$. bovienii belonging to $S$. feltiae.

\section{DISCUSSION}

Our results suggested that Xenorhabdus spp. may encode a plethora of functions not directly related to their role as endosymbionts of Steinernematidae, and a considerable phenotypic variability is present among strains of $X$. bovienii that could not be explained solely on the basis of guest-host association.

In fact those organisms are very similar to other Enterobacteriaceae, are able to grow in several media (both synthetic and natural) and might also exchange genetic material. Their genomes are large (4 to $5 \mathrm{Mb}$ and may be more), suggesting that the association with Steinernema spp., although specific, has not hampered the full functionality of the genome. Congruent with this view is the pattern of biofilm production, which seems very heterogeneous in $X$. bovienii but more conserved in the $X$. kozodoii strains. For this reason, we raised the hypothesis of neutrality for most of the characters chosen for analysis.

The composite pattern of antibiotic resistance/susceptibility seems very puzzling. Resistance to $\beta$-lactams, confirmed by our assays, was already described for almost all Xenorhabdus species. Genes contributing resistance to $\beta$ lactames have already been found in $X$. bovienii and $X$. nematophila genomes. However, the pattern of resistance to other antibiotics, in absence of any obvious exposure, is generally composite and suggests an acquisition of resistance genes from the environment. A suitable approach to answer that question will imply the identification of the resistance genes and the elucidation of the resistance mechanism(s). It has been shown that the genome of $X$. bovienii still contains mobilizing elements (BISCH et al., 2016), suggesting those endosymbionts to be able to assume and mobilize DNA and/or genetic elements from and to the environment. More work is needed to elucidate the mechanisms behind such intriguing behaviour of endosymbionts.

It has been suggested that toxins produced by Xenorhabdus spp. should be considered as a selectable marker, because each strain competes with any other in case of coinfection (ADAMS et al., 2007; BlouIN et al., 1999). Our results showed that this is the case even at the doses used; moreover, the toxin preparation was able to kill even larvae so large as those of Rhynchophorus ferrugineus, independently of the strain used (unpublished data). Our protocol was adapted from that of BRILLARD et al. (2001); those authors were able to purify a toxin from $X$. nematofila, which was later identified as the product of the $x a x A B$ gene system (VIGNEUX et al., 2007). However, since that genetic system is absent in the genome of $X$. bovienii, it is conceivable that other toxins, with similar physicochemical properties, are produced by other Xenorhabdus species. Different toxins, or their proportions, might be responsible for the differential response of $G$. mellonella larvae in our experiments. However, because larval death was achieved within 24 hrs post injection regardless of the strain, our observations do not provide evidence of actual differences in virulence between strains. More experiments are needed to clarify the role of these bacterial endosymbionts in the biological control action of the entomopathogenic nematodes to which they are associated.

\section{ACKNOWLEDGEMENTS}

Research partially funded by Annual Research Plan 201618 of Dept. Biological Geological Environmental Sciences, University of Catania (grants \# 22722132110 and 22722132113). Further, the authors wish to thank dr. Fabio Viglianisi for help with SIMCA. 


\section{REFERENCES}

Adams B.J., Peat S.M., Dillman A.R., 2007 - Chapter 6: Phylogeny and Evolution. In: Entomopathgenic nematodes: Systematics, Phylogeny and bacterial symbionts". - Nematology Monographs and perspectives Vol 5. Nguyen \& Hunt Eds. (Brill Leiden-Boston 2007): 693-733.

Bisch G., Ogier J.-C., Médigue C., Rouy Z., Vincent S., Tailliez P., Givaudan A., Gaudriault S., 2016 Comparative Genomics between Two Xenorhabdus bovienii Strains Highlights Differential Evolutionary Scenarios within an Entomopathogenic Bacterial Species. - Genome Biology and Evolution, 8 (1): 148-160.

Blouin M.S., LiU J., Berry R.E., 1999 - Life cycle variation and the genetic structure of nematode populations. - Heredity, 83: 253-259.

Brillard J., Ribeiro C., Boemare N., Brehèlin M., Givaudan A, 2001 - Two Distinct Hemolytic Activies in Xenorhabdus nematophila Are Active against Immunocompetent Insect Cells. - Applied and Enviromental Microbiology, 67(6): 2515-2525.

Clausi M., Rappazzo G., Vinciguerra, M.T., 2012 - The complex and multiform relationship between nematoda and bacteria. - Redia, 95: 79-82.

Clausi M., Leone D., Vinciguerra M.T., Rappazzo G., TARASCO E., 2014 - Laboratory tests on the biocontrol of chestnut Insect pests on Etna (Sicily, Italy) by means of entomopathogenic nematodes. - Redia, 97: 133-136.

Clausi M., Longo A., Rappazzo G., Tarasco E., VINCIGUERRA M.T., 2011 - Steinernema vulcanicum $n$. sp. (Rhabditida: Steinernematidae), a new entomopathogenic nematode species from Sicily (Italy). Nematology, 13(4): 409-423.

Clinical and Laboratory Standards Institute (CLSI), 2017 - Performance Standards for Antimicrobial Susceptibility Testing. 27 $7^{\text {th }} \mathrm{ed}$. Clinical and Laboratory Standards Institute (CLSI); Wayne, PA, USA: 2017. CLSI Supplement M100S.

De Luca F., Clausi M., Troccoli A., Curto G., Rappazzo G., TARASCO E., 2015 - Entomopathogenic nematodes in Italy: occurrence and use in microbial control strategies. - In: Nematode pathogenesis of insects and other pests, 431-449.

Fuochi V., Li Volti G., Furneri M.P., 2017 - Probiotic properties of Lactobacillus fermentum strains isolated from human oral samples and description of their antibacterial activity. - Current pharmaceutical biotechnology, 18(2): 138-149.
Giummarra V., Scuderi M.C., Tempera G., Roccasalva L.S., Furneri P.M., 2010 - Relationships between hydrophobicity and biofilm formation in Streptococcus agalactiae strains. - In: Microrganisms in industry and environment: 192-194

KOPPENHOFER H.S., 2007 - Chapter 7: Bacterial symbionts of Steinernema and Heterorhabditis. In "Entomopathgenic nematodes: Systematics, Phylogeny and bacterial symbionts", Nematology Monographs and perspectives Vol 5. Nguyen \& Hunt Eds. (Brill LeidenBoston 2007): 735-808

Hinchliffe S.J., Hares M.C., Dowling A.J., FFrenchConstant R.H., 2010 - Insecticidal Toxins from the Photorhabdus and Xenorhabdus Bacteria. - The Open Toxicology Journal, 3: 83-100.

McKay L., Miller A. 3RD, Sandine W.E., Elliker P.R., 1970 - Mechanisms of lactose utilization by lactic acid streptococci: enzymatic and genetic analyses. - J Bacteriol., 102(3): 804-809.

PoINAR G.O. JR., 1979 - Nematodes for Biological Control of Insects. CRC Press, Boca Raton, Fl.: 277 pp.

PoINAR G.O. JR., 1990 - Taxonomy and biology of Steinernematidae and Heterorhabditidae. In: Entomopathogenic Nematodes in Biological Control, Gaugler R.D. \& Kaya H.K. Ed., Boca Raton FL. USA. CRC Press, pp. 23-61.

Tailliez P., Pagès S., Ginibre N., Boemare N. 2006 - New insight into diversity in the genus Xenorhabdus, including the description of ten novel species. - International Journal of Systematic and Evolutionary Microbiology, 56: 2805-2818.

Tarasco E., Clausi M., Rappazzo G., Panzavolta T., Curto G., Sorino R., Oreste M., Longo A., Leone D., Tiberi R., Vinciguerra M.T., Triggiani O., 2015 Biodiversity of entomopathogenic nematodes in Italy. Journal of Helminthology 89(3): 359-366.

Tarasco E., Mracek Z., Nguyen K.B., Triggiani O., 2008 - Steinernema ichnusae sp. n. (Nematoda: Steinernematidae) a new entomopathogenic nematode from Sardinia Island (Italy). - J. Invertebr. Pathol., 99(2): 173185.

Vigneux F., Zumbinl R., Jubelin G., Ribeiro C., Poncet J., Baghdiguian S., Givaudan A., BrehÉLIN M., 2007 - The xaxAB Genes Encoding a New Apoptotic Toxin from the Insect Pathogen Xenorhabdus nematophila Are Present in Plant and Human Pathogens. - The Journal of Biological Chemistry, 282 (13): 9571-9580. 